\title{
A Novel Environment Exploration Strategy by m-generalised q-neutrosophic WASPAS
}

\author{
Rokas SEMENAS ${ }^{1}$, Romualdas BAUSYS ${ }^{1 *}$, Edmundas Kazimieras ZAVADSKAS ${ }^{2}$ \\ ${ }^{1}$ Vilnius Gediminas Technical University, Department of Graphical Systems, \\ Sauletekio Ave. 11, LT-10223 Vilnius, Lithuania \\ rokas.semenas@vgtu.lt, romualdas.bausys@vgtu.lt (*Corresponding author) \\ ${ }^{2}$ Vilnius Gediminas Technical University, Institute of Sustainable Construction, \\ Sauletekio Ave. 11, LT-10223 Vilnius, Lithuania \\ edmundas.zavadskas@vgtu.lt
}

\begin{abstract}
Unknown environment exploration by an autonomous robot is a complex problem that requires robustness and reliability of the applied exploration strategy. Currently, a common approach to autonomous exploration is to incrementally increase the robot's knowledge about the environment by directing it to the regions which border currently unexplored areas (frontiers). However, deciding where to move next when multiple alternatives are present introduces an additional layer of complexity. As such, a decision might require balancing the competing high-level objectives (for example, visiting several priority locations while also reducing the robot travelled distance). This research proposes a novel environment exploration strategy and the extension for the WASPAS multi-criteria decision making (MCDM) method, modelled under the m-generalised q-neutrosophic environment, namely, WASPAS-mGqNS. The proposed method is applied to address the problem of selecting the next frontier that the exploring robot should reach. Case study results highlight how the proposed approach could be applied to minimise the robot-travelled distance and maximise the observed environment when the robot is tasked to visit several priority locations set in advance by the robot operator.
\end{abstract}

Keywords: Multi-criteria decision making, m-generalised q-neutrosophic sets, WASPAS-mGqNS, Autonomous environment exploration.

\section{Introduction}

Multi-criteria decision making (MCDM) methods are exceptional tools that can be applied in many real-world decision problems that require determining the optimal alternative when considering several competing requirements (Aruldoss, Lakshmi \& Venkatesan, 2013). However, incomplete or uncertain input data characteristics are a prominent issue that causes researchers to look for modern techniques when modelling such data in complex decision-making problems.

In particular, the fuzzy set theory (Zadeh, 1965) is considered an effective technique to model input data characteristics. Here, a single input object $x$ is modelled as a value $\mu(x) \in[0,1]$ that represents its membership degree to the object universe $X$ (Wang et al., 2010). However, the classical fuzzy sets are limited when considering the decision-making process with incomplete information. As it is not always true that the sum is equal to the one between the membership and non-membership degrees, the Intuitionistic fuzzy sets were introduced by Atanassov (1986) as the generalised fuzzy set which incorporated the hesitation degree. Techniques based on fuzzy set theory were further extended when Pythagorean fuzzy sets (Yager, 2013) were presented as another approach of modelling imprecise membership degrees, and the q-Rung orthopair fuzzy sets
(Yager, 2017) were introduced to increase the space of the acceptable membership values.

In 1999, the neutrosophic sets were introduced by Smarandache (1999) as the generalisation of classical fuzzy and intuitionistic fuzzy sets. By applying neutrosophic logic, each input data parameter is defined by the independent truth membership degree - $\mathrm{T}$, indeterminacy membership degree - I, and falsity membership degree $-\mathrm{F}$. The inclusion of the indeterminacy component (also defined as unknown (Smarandache, 2006)) and the ability to model these memberships independently distinguishes the neutrosophic set from other sets applied to model the characteristics of input data. Due to these advantages, neutrosophic sets were successfully utilised in multiple MCDM problems, where the vagueness of input data had to be considered (e.g., (Zavadskas et al., 2020b; Zavadskas et al., 2021), etc.).

However, as highlighted in the recent paper by Smarandache (2019), the neutrosophic set is actually the generalisation of the Intuitionistic fuzzy set, Pythagorean fuzzy set, q-Rung orthopair fuzzy set, Spherical and n-Hyper Spherical fuzzy sets. Thus, these sets can be combined under one m-generalised q-neutrosophic set (mGqNS), which could be suitable for modelling a flexible 
decision-making framework for complex realworld problems (Zavadskas et al., 2020a).

One of such problems, in particular, is the decision-making by the autonomous robot, which is deployed to explore the unknown (or known, but structurally changed) environment. Currently, environment exploration strategies are commonly based on increasing the knowledge about the environment properties by moving the robot to the boundaries between the explored and unknown space (Juliá, Gil \& Reinoso, 2012), called frontiers (Yamauchi, 1997). By following this approach, every time a robot visits a frontier and adds new information to the partial environment map, the list of available frontiers is updated, and the robot makes a new decision of where to move next.

The complexity of this decision-making task comes from uncertainties and incomplete input data under which the decisions of how to navigate in the environment must be made to balance the competing high-level objectives. For example, robot operators might want the robot to explore a specific location, maximise the covered area to construct a representative environment map, and minimise the travelled distance - all with limited interactions from human operators (Calisi et al., 2007; Akin et al., 2013). Thus, frontier-based exploration strategies differ in terms of how the frontiers are assessed according to the given high-level objective. For example, the robot can be sent to the closest frontier to cover the whole exploration space (Yamauchi, 1997) or it can be directed to heat signatures to help identify humans (Cakmak et al., 2017). Also, MCDM methods can be applied to combine multiple criteria, such as the length of the shortest path to the frontier, the amount of unknown space behind the frontier, and the ability to communicate with the robot control station after reaching the frontier (Basilico \& Amigoni, 2011; Taillandier \& Stinckwich, 2011) to determine the utility of a frontier according to the high-level objective.

However, the strategies above are applied considering situations where no initial information about the exploration environment is known in advance, and the robot is tasked to cover most of the exploration space. Yet, considering some real-world scenarios (e.g., search and rescue missions or evacuation route planning (Chandrawati, Ratna \& Sari, 2020)), it is very likely that robot operators can gather a-priori information about the environment and utilise it to direct the exploration process accordingly (Calisi et al., 2007; Roa-Borbolla, 2017). Thus, in this research, a novel approach to frontier assessment and decision-making problem for an autonomous exploring robot is proposed. Unlike in previous studies, the proposed strategy enables the robot to explore areas around the priority locations and reduce the amount of input data needed to filter the frontiers surrounded by mostly explored space. This strategy's main goal is to maximise the covered area around a set of priority locations while minimising the robot travelled distance. Also, a novel extension of the state-of-theart WASPAS method (which was originally proposed by Zavadskas et al., (2012)), modelled under the m-generalised q-neutrosophic environment is introduced, namely, WASPASmGqNS. This novel MCDM method can provide additional flexibility for robot operators when considering uncertain or incomplete input data in environment exploration missions.

This research is organised as follows. The proposed frontier assessment strategy is presented in Section 2. The algebraic operations of m-generalised q-neutrosophic sets and the proposed WASPAS-mGqNS method are discussed in Section 3. Section 4 represents the case study and the computational example of the frontier assessment process. Finally, conclusions are presented in Section 5.

\section{Frontier Assessment Strategy}

In this research, three environment exploration strategies are considered: a direct control strategy (further referred to as WS), in which the robot follows the shortest path between the priority locations, visiting them in the order specified by the robot operator; information gain strategy (further referred to as IG), which utilises technical frontier assessment criteria (e.g., estimated time that is needed to reach the frontier, or distance to the candidate frontier) derived from (Basilico \& Amigoni, 2011; Taillan dier \& Stinckwich, 2011); and the proposed strategy (further referred to as PS) which expands the IG strategy by addressing the problem of visiting priority locations, specified by the robot operator. 
Table 1. Criteria set applied to model frontier assessment strategies

\begin{tabular}{|c|c|c|c|c|}
\hline ID & Optimum & Criteria name & PS & IG \\
\hline$C_{1}$ & Min & $\begin{array}{l}\text { Distance to } \\
\text { the candidate } \\
\text { frontier. }\end{array}$ & 0.07 & 0.25 \\
\hline$C_{2}$ & Max & $\begin{array}{c}\text { Expected } \\
\text { information gain. }\end{array}$ & 0.13 & 0.30 \\
\hline$C_{3}$ & Min & $\begin{array}{l}\text { Estimated time } \\
\text { needed to reach } \\
\text { the frontier. }\end{array}$ & 0.24 & 0.35 \\
\hline$C_{4}$ & Min & $\begin{array}{l}\text { Distance to the } \\
\text { robot control } \\
\text { station. }\end{array}$ & 0.04 & 0.10 \\
\hline$C_{5}$ & Min & $\begin{array}{l}\text { Distance from the } \\
\text { frontier to the set } \\
\text { priority location. }\end{array}$ & 0.37 & - \\
\hline$C_{6}$ & Max & $\begin{array}{l}\text { Sampling ratio } \\
\text { around the } \\
\text { frontier. }\end{array}$ & 0.15 & - \\
\hline
\end{tabular}

The criteria used by IG and PS strategies are presented in Table 1, together with their optimums and relative weights, which were determined by several experts working in the field of MCDM and autonomous robots. In the present setup, the grid map environment representation model is used for frontier detection and the estimation of some criteria values.

- $C_{1}$, the distance to the candidate frontier is measured by the Euclidean distance between the current robot location and the candidate frontier. The criterion is minimised to prioritise frontiers located close to the robot to reduce backtracking behaviour;

- $\quad C_{2}$, the expected information gain is considered to be equal to the frontier length and is maximised to direct the robot to frontiers which are assumed to border wide open spaces;

- $C_{3}$, the estimated time needed to reach the frontier is applied to prioritise the frontiers close to the robot and reachable by short and straight paths. To estimate the criterion value, the methodology defined by Basilico \& Amigoni (2011), which was also applied in our previous robot setup (Semenas \& Bausys, 2020), is applied in this case. The constant average robot movement and rotation velocities of $0.4 \mathrm{~m} / \mathrm{s}$ and $0.5 \mathrm{rad} / \mathrm{s}$ are used in this research;

- $\quad C_{4}$, the distance to the robot control station is measured as the Euclidean distance between the assessed frontier and the robot starting position. This criterion is minimised to ensure the prioritisation of frontiers located closer to the control station, enabling the robot to transmit the acquired data to robot operators;

- $C_{5}$, the distance from the frontier to the set priority location is a minimised criterion that is introduced to enable more exhaustive exploration around the set priority location without directly moving the robot to it. In a sense, this approach can be used to focus exploration effort on the prioritised locations, similarly as in (Roa-Borbolla et al., 2017), thus reducing the time needed to detect important information. The criterion value is measured by the shortest Euclidean distance between each priority location and the assessed frontier. It is also worth noting that due to the generality of the MCDM approach, even in situations where no priority locations are set, the robot will explore the environment by applying the remaining $C_{1}-C_{4}$ and $C_{6}$ criteria. However, in such cases, to address the specifics of neutrosophic sets, the default $C_{5}$ criteria values should be set to a small positive constant;

- $\quad C_{6}$, the sampling ratio around the frontier is a maximised criterion that is introduced to reduce the chance of selecting frontiers that are unreachable or are mostly surrounded by already explored space (e.g., frontiers that are located in the corners or near walls). Also, this criterion is applied to address and somewhat mitigate the issue of incomplete or noisy robot-constructed grid map, as this problem can significantly influence the autonomous robot performance (Zakiev et al., 2019). For example, some frontiers may be detected in the middle of the wall due to the faulty sensor data and incorrect cell occupancy estimation of the used grid map;

In this research, the criteria value is determined by sampling a total of 100 cells 
within a set radius around each frontier as presented in Figure 1. In this setup, the radius value is equal to $1.5 \mathrm{~m}$. The blue line indicates the frontier, and the green marker indicates its centre point. The red markers indicate sample points that are located on the explored or occupied space. Yellow markers indicate sample points that are located in the undiscovered space. The criterion value is assessed by applying the following equation:

$$
C_{6}=\frac{\delta}{\lambda \cdot 2^{n}}
$$

where $\delta$ is the number of sampled cells that are yet to be discovered, $\lambda$ is the sample population size and $n$ is the number of sampled cells that are occupied.

Although the approach of estimating the amount of free space that would be visible by considering robot perception sensor parameters can also be applied to determine the value of this criterion, this approach requires the computation of more input data.

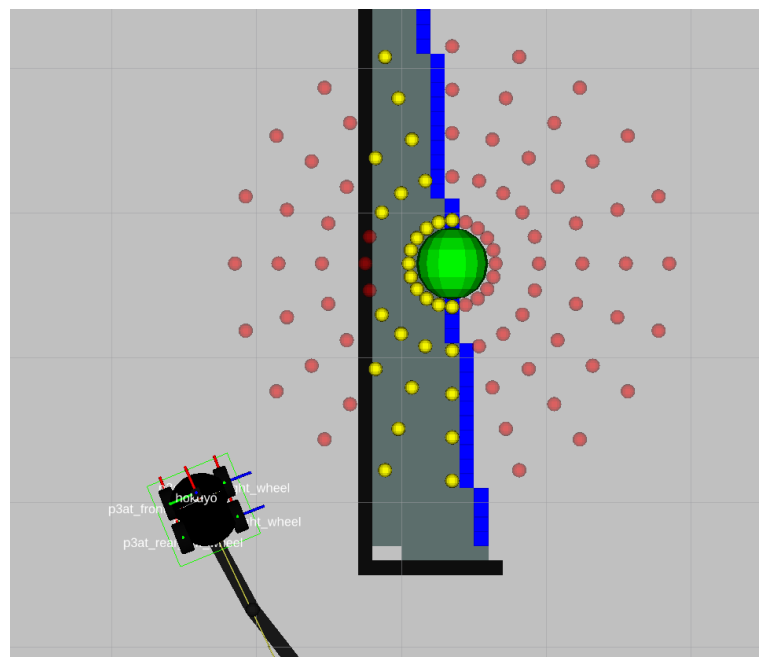

Figure 1. Approach for cell sampling around the frontier

Next, the concepts of m-generalised q-neutrosophic set and the novel WASPASmGqNS method are introduced.

\section{Preliminaries of m-generalised q-neutrosophic Set}

First, the preliminaries of $\mathrm{m}$-generalised q-neutrosophic set (mGqNS) and the algebraic operations between the $\mathrm{m}$-generalised q-neutrosophic numbers (mGqNNs) that are relevant to the proposed m-generalised q-neutrosophic WASPAS method, namely, WASPAS-mGqNS, are discussed.

3.1 Definition. If the domain of problem-related objects is denoted by $X, x \in X$ is a single object. In this research, $X$ is a set of criteria modelled under the m-generalised q-neutrosophic environment and $x$ is a value of a single criterion.

Let $q \geq 1$, and $m=1 \| 3$. The m-generalised q-neutrosophic set $A_{m q}$ is defined by the three membership functions:

$T_{m q}, I_{m q}, F_{m q}: X \rightarrow[0, r],(0 \leq r \leq 1)$

Here $T_{m q}$ is the m-generalised truth membership function, $I_{m q}$ is the m-generalised indeterminacy membership function, and $F_{m q}$ is the m-generalised falsity membership function. The m-generalised q-neutrosophic set is defined as:

$A_{m q}=\left\{\left\langle T_{m q}(x), I_{m q}(x), F_{m q}(x)\right\rangle: x \in X\right\}$

The three membership functions also satisfy the following conditions:

$0 \leq T_{m q}(x), I_{m q}(x), F_{m q}(x) \leq 1$ for every $x \in X$;

$0 \leq\left(T_{m q}(x)\right)^{q}+\left(I_{m q}(x)\right)^{q}+\left(F_{m q}(x)\right)^{q} \leq \frac{3}{m}$ for every $x \in X$;

Note that different $m$ and $q$ values define different neutrosophic sets.

3.2 Definition. A m-generalised q-neutrosophic number, mGqNN, is defined as $N_{m q}=\langle t, i, f\rangle$.

3.3 Definition. Let $N_{m q_{1}}=\left\langle t_{1}, i_{1}, f_{1}\right\rangle$ and $N_{m q_{2}}=\left\langle t_{2}, i_{2}, f_{2}\right\rangle$ be two m-generalised q-neutrosophic numbers. Then the summation of the two $\mathrm{mGqNNs}$ can be defined by:

$N_{m q_{1}} \oplus N_{m q_{2}}=\left\langle\left(1-\left(1-t_{1}^{q}\right)\left(1-t_{2}^{q}\right)\right)^{\frac{1}{q}}\right.$,

$\left.i_{1} i_{2}, f_{1} f_{2}\right\rangle$

The multiplication between the two mGqNNs can be defined by:

$$
\begin{aligned}
& N_{m q_{1}} \otimes N_{m q_{2}}=\left\langle t_{1} t_{2},\left(1-\left(1-i_{1}^{q}\right)\left(1-i_{2}^{q}\right)\right)^{\frac{1}{q}},\right. \\
& \left.\left(1-\left(1-f_{1}^{q}\right)\left(1-f_{2}^{q}\right)\right)^{\frac{1}{q}}\right\rangle
\end{aligned}
$$


The multiplication operation between the $\mathrm{mGqNN}$ and a real number $\lambda>0$ can be defined by:

$$
\lambda \cdot N_{m q_{1}}=\left\langle\left(1-\left(1-t_{1}^{q}\right)^{\lambda}\right)^{\frac{1}{q}}, i_{1}^{\lambda}, f_{1}^{\lambda}\right\rangle
$$

The power function of $\mathrm{mGqNN}$ when $\lambda>0$ can be defined by:

$$
\begin{aligned}
& \lambda \odot N_{m q_{1}}= \\
& \left\langle t_{1}^{\lambda},\left(1-\left(1-i_{1}^{q}\right)^{\lambda}\right)^{\frac{1}{q}},\left(1-\left(1-f_{1}^{q}\right)^{\lambda}\right)^{\frac{1}{q}}\right\rangle
\end{aligned}
$$

To ensure the stability of the neutrosophic set logic, the complementary function of $\mathrm{mGqNN}$ can be defined by:

$$
N_{m q}{ }^{c}=\left\langle f_{1}, 1-i_{1}, t_{1}\right\rangle
$$

3.4 Definition. The score value $S\left(N_{m q}\right)$ can be determined by:

$$
S\left(N_{m q}\right)=\frac{3+3 t^{q}-2 i^{q}-f^{q}}{6}
$$

If $N_{m q_{1}}=\left\langle t_{1}, i_{1}, f_{1}\right\rangle$ and $N_{m q_{2}}=\left\langle t_{2}, i_{2}, f_{2}\right\rangle$ are two m-generalised q-neutrosophic numbers, the ranking of them is performed by:

$$
\begin{aligned}
& \text { If } S\left(N_{m q_{1}}\right)>S\left(N_{m q_{2}}\right) \text {, then } N_{m q_{1}}>N_{m q_{2}} \\
& \text { If } S\left(N_{m q_{1}}\right)=S\left(N_{m q_{2}}\right) \text {, then } N_{m q_{1}}=N_{m q_{2}}
\end{aligned}
$$

\subsection{WASPAS-mGqNS Method}

In this research, the state-of-the-art WASPAS method is extended by modelling it under the m-generalised q-neutrosophic environment. In its general form, the WASPAS-mGqNS method can be defined by the following steps:

Step 1. The decision matrix $D_{m q}$ is constructed from a set of available alternatives (in this research - frontiers) in accordance to the task-related criteria. Arrays of the decision matrix can be denoted by $\left[d_{m q}\right]_{i j}$, where $i=1, \ldots, k$ and $j=1, \ldots, n$ are the frontier and criteria indexes, respectively. In this case, $\left[d_{m q}\right]_{i j}$ represents the value of the $i^{\text {th }}$ frontier according to the $j^{\text {th }}$ criterion;

Step 2. The vector normalisation approach, that was developed for appropriate estimation of the certain features of the neutrosophic sets (Zavadskas et al., 2020a), is applied to normalise the arrays of the decision matrix:

$$
\left[\bar{d}_{m q}\right]_{i j}=\frac{\left[d_{m q}\right]_{i j}}{\sqrt{\sum_{s=1}^{n}\left(\left[d_{m q}\right]_{s j}\right)^{2}}}
$$

Step 3. Next, the neutrosophication of the normalised decision matrix $\bar{D}_{m q}$ is conducted by applying the neutrosophication table, as in (Zavadskas et al., 2020b). After this step, $\left[\bar{d}_{m q}\right]_{i j}$ arrays of the decision matrix $\bar{D}_{m q}$ obtain the general mGqNN form of $\left[\bar{d}_{m q}\right]_{i j}=\left\langle t_{i j}, i_{i j}, f_{i j}\right\rangle$ (as in 3.2 definition);

Step 4. The first objective of $\mathrm{m}$-generalised q-neutrosophic WASPAS is the computation of total relative importance of the alternatives (frontiers), which is obtained by applying the following function:

$Q_{i}^{(1)}=\sum_{j=1}^{O_{\max }}\left[\bar{d}_{m q}\right]_{i j} \cdot w_{j}+\left(\sum_{j=1}^{O_{\min }}\left[\bar{d}_{m q}\right]_{i j} \cdot w_{j}\right)^{c}$

Here, $O_{\max }$ and $O_{\min }$ represent the maximised and the minimised arrays of $\bar{D}_{m q}, w_{j}$ represents the weight of criterion $C_{j}$, defined by a real number $w_{j}>0$, and $c$ indicates a complementary neutrosophic number;

Step 5. The second objective of the WASPAS$\mathrm{mGqNS}$ method is the product of the total relative importance of the alternatives (frontiers), which is obtained by applying the following function:

$$
Q_{i}^{(2)}=\prod_{j=1}^{O_{\max }}\left(\left[\bar{d}_{m q}\right]_{i j}\right)^{w_{j}} \cdot\left(\prod_{j=1}^{O_{\min }}\left(\left[\bar{d}_{m q}\right]_{i j}\right)^{w_{j}}\right)^{c}
$$

Here, the component definitions are identical to the ones provided for Equation (9). The algebraic operations that are applied in both objectives of the WASPAS-mGqNS method are provided in Section 3 of this paper;

Step 6. The joint generalised value that incorporates the results obtained from step 4 and step 5 is determined by:

$Q_{i}=0.5 Q_{i}^{(1)}+0.5 Q_{i}^{(2)}$

Step 7. Lastly, frontier rankings are determined by utilising a score function $S\left(Q_{i}\right)$ and the highestranked alternative (frontier) is considered to be the next robot goal.

\section{Case Study}

To highlight how the proposed environment exploration strategy PS and the WASPAS- 
mGqNS method could be applied in autonomous environment exploration tasks, they are tested in a Gazebo-simulated indoor environment, presented in Figure 2. The yellow circles mark priority locations that the robot should visit. The blue square marks the robot starting position. The main goal of the proposed environment exploration strategy is to minimise the robot travelled distance while also increasing the amount of covered area around a set of priority locations that human operators identify before deploying the autonomous robot.

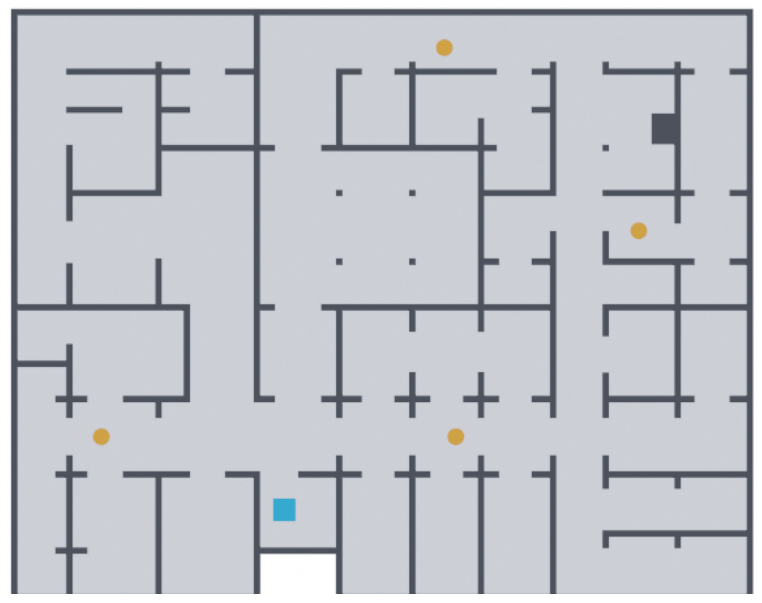

Figure 2. Simulated indoor environment

The autonomous robot deployed in a Gazebo simulator is controlled by utilising the robot operating system (ROS) and using a similar navigation framework and sensor setup as the one introduced in our previous research (Semenas $\&$ Bausys, 2020). The robot utilises the frontierbased environment exploration approach. The decision of where to move next is made, and the highest-ranked frontier is determined by applying the proposed WASPAS-mGqNS method. In the present setup, the exploration process is completed when all four priority locations are visited by the robot or the time limit of 10 minutes is reached (here, the movement time is used, assuming that the robot battery is drained at a constant speed while the robot is moving). Also, some reasonable additional assumptions are made:

- It is assumed that the robot operator has information that allows $\mathrm{him} / \mathrm{her}$ to set the coordinates of priority locations that the robot should visit. However, no information about the current state of the environment is known to the robot or the operator in advance;

- Autonomous robot must cancel its current task and reach the prioritised location if it is nearby. The prioritised location is considered as visited when the distance between it and the current robot position is less than $1.5 \mathrm{~m}$;

- Autonomous robot is able to change its navigation goal if a higher-valued frontier is detected while moving to the previously selected frontier;

- No additional hazards or moving objects that could damage the robot are present in the environment.

The three previously introduced strategies - PS, IG, and WS, are considered in this case study. Here, PS and IG strategies follow the frontier-based environment exploration approach by applying the WASPAS-mGqNS method and frontier assessment strategies introduced in Table 1. The WS strategy assumes the direct control approach, where the robot operator sets the order in which priority locations should be visited, and the robot follows the shortest path between them.

Next, a computational example is provided to highlight how the proposed WASPAS-mGqNS method can be applied for frontier assessment in an autonomous environment exploration task.

\subsection{Computational Example and Case Study Results}

In this example, one of the environment exploration iterations is considered when the robot is applying the proposed PS strategy for frontier assessment. The values of $m=1$ and $q=3$ are chosen for the m-generalised q-neutrosophic numbers. In the considered environment exploration state, 11 frontiers are assessed by applying the proposed WASPAS-mGqNS method.

The crisp sensor data values, which are obtained during robot navigation and used to construct the initial decision_matrix $D_{m q}$, are presented in Table 2 . The $\left[\bar{d}_{m q}\right]_{i j}$ values obtained after the normalisation and the neutrosophication of the initial decision matrix are presented in Table 3. Next, the two objectives of WASPASmGqNS method are calculated by applying the m-generalised q-neutrosophic algebra (Section 3). The final rankings of the candidate frontiers in the considered decision-making iteration are determined by applying the score function (Equation (7)) and presented in Table 4. In this example, the frontier denoted as $P_{2}$ is considered as the optimal location for further environment 
Table 2. The initial decision matrix values for frontier assessment problem in a sample decision-making iteration.

\begin{tabular}{|c|c|c|c|c|c|c|c|c|c|c|c|}
\hline & $P_{1}$ & $P_{2}$ & $P_{3}$ & $P_{4}$ & $P_{5}$ & $P_{6}$ & $P_{7}$ & $P_{8}$ & $P_{9}$ & $P_{10}$ & $P_{11}$ \\
\hline$C_{1}$ & 3.20 & 4.22 & 6.64 & 10.42 & 6.15 & 12.02 & 11.29 & 12.36 & 18.41 & 14.80 & 18.50 \\
\hline$C_{2}$ & 2.9 & 3.5 & 3.6 & 10.8 & 2.0 & 6.7 & 4.6 & 4.6 & 5.7 & 1.5 & 5.7 \\
\hline$C_{3}$ & 11.99 & 13.59 & 20.02 & 29.71 & 19.43 & 33.02 & 31.54 & 34.95 & 48.96 & 42.09 & 49.59 \\
\hline$C_{4}$ & 3.38 & 4.27 & 6.78 & 10.54 & 6.33 & 12.17 & 11.45 & 12.46 & 18.56 & 14.97 & 18.66 \\
\hline$C_{5}$ & 6.05 & 5.36 & 8.25 & 8.81 & 6.11 & 10.96 & 9.83 & 8.66 & 7.59 & 6.88 & 5.16 \\
\hline$C_{6}$ & 0.51 & 0.53 & 0.46 & 0.50 & 0.27 & 0.52 & 0.50 & 0.51 & 0.52 & 0.59 & 0.51 \\
\hline
\end{tabular}

Table 3. The constructed neutrosophic matrix for frontier assessment problem in a sample decision-making iteration

\begin{tabular}{|c|c|c|c|c|c|c|c|c|c|c|c|}
\hline & $P_{1}$ & $P_{2}$ & $P_{3}$ & $P_{4}$ & $P_{5}$ & $P_{6}$ & $P_{7}$ & $P_{8}$ & $P_{9}$ & $P_{10}$ & $P_{11}$ \\
\hline \multirow{4}{*}{$C_{1}$} & $(0.08$, & $(0.11$, & $(0.17$, & $(0.27$, & $(0.16$, & $(0.31$, & $(0.29$, & $(0.31$, & $(0.47$, & $(0.38$, & $(0.47$, \\
& 0.92, & 0.90, & 0.87, & 0.78, & 0.87, & 0.74, & 0.76, & 0.74, & 0.55, & 0.67, & 0.54, \\
& $0.92)$ & $0.89)$ & $0.83)$ & $073)$ & $0.84)$ & $0.69)$ & $0.71)$ & $0.69)$ & $0.53)$ & $0.62)$ & $0.53)$ \\
\hline \multirow{4}{*}{$C_{2}$} & $(0.17$, & $(0.20$, & $(0.20$, & $(0.61$, & $(0.11$, & $(0.38$, & $(0.26$, & $(0.26$, & $(0.32$, & $(0.09$, & $(0.32$, \\
& 0.87, & 0.85, & 0.85, & 0.34, & 0.89, & 0.67, & 0.79, & 0.79, & 0.73, & 0.91, & 0.73, \\
& $0.83)$ & $0.80)$ & $0.80)$ & $0.39)$ & $0.89)$ & $0.62)$ & $0.74)$ & $0.74)$ & $0.68)$ & $0.91)$ & $0.68)$ \\
\hline \multirow{4}{*}{$C_{3}$} & $(0.11$, & $(0.12$, & $(0.18$, & $(0.27$, & $(0.18$, & $(0.30$, & $(0.29$, & $(0.32$, & $(0.45$, & $(0.39$, & $(0.45$, \\
& 0.90, & 0.89, & 0.86, & 0.78, & 0.86, & 0.75, & 0.76, & 0.73, & 0.58, & 0.66, & 0.57, \\
& $0.89)$ & $0.88)$ & $0.82)$ & $0.73)$ & $0.82)$ & $0.70)$ & $0.71)$ & $0.68)$ & $0.55)$ & $0.61)$ & $0.55)$ \\
\hline \multirow{4}{*}{$C_{4}$} & $(0.09$, & $(0.11$, & $(0.17$, & $(0.27$, & $(0.16$, & $(0.31$, & $(0.29$, & $(0.31$, & $(0.47$, & $(0.38$, & $(0.47$, \\
& 0.91, & 0.90, & 0.86, & 0.78, & 0.87, & 0.74, & 0.76, & 0.74, & 0.55, & 0.67, & 0.55, \\
& $0.91)$ & $0.89)$ & $0.83)$ & $0.73)$ & $0.84)$ & $0.69)$ & $0.71)$ & $0.69)$ & $0.53)$ & $0.62)$ & $0.53)$ \\
\hline \multirow{4}{*}{$C_{5}$} & $(0.23$, & $(0.21$, & $(0.32$, & $(0.34$, & $(0.24$, & $(0.42$, & $(0.38$, & $(0.33$, & $(0.29$, & $(0.27$, & $(0.20$, \\
& 0.82, & 0.84, & 0.73, & 0.71, & 0.81, & 0.62, & 0.67, & 0.72, & 0.76, & 0.78, & 0.74, \\
& $0.77)$ & $0.79)$ & $0.68)$ & $0.66)$ & $0.76)$ & $0.58)$ & $0.62)$ & $0.67)$ & $0.71)$ & $0.73)$ & $0.80)$ \\
\hline \multirow{4}{*}{$C_{6}$} & $(0.31$, & $(0.32$, & $(0.28$, & $(0.30$, & $(0.16$, & $(0.31$, & $(0.30$, & $(0.31$, & $(0.31$, & $(0.36$, & $(0.31$, \\
& 0.74, & 0.73, & 0.77, & 0.75, & 0.87, & 0.74, & 0.74, & 0.74, & 0.74, & 0.69, & 0.74, \\
& $0.69)$ & $0.68)$ & $0.72)$ & $0.70)$ & $0.84)$ & $0.69)$ & $0.70)$ & $0.69)$ & $0.69)$ & $0.64)$ & $0.69)$ \\
\hline
\end{tabular}

Table 4. The results of WASPAS-mGqNS method for a sample decision-making iteration

\begin{tabular}{|c|c|c|c|c|c|c|c|c|c|c|c|}
\hline & $P_{1}$ & $P_{2}$ & $P_{3}$ & $P_{4}$ & $P_{5}$ & $P_{6}$ & $P_{7}$ & $P_{8}$ & $P_{9}$ & $P_{10}$ & $P_{11}$ \\
\hline $0.5 Q^{(1)}$ & $\begin{array}{c}(0.72, \\
0.34, \\
0.40)\end{array}$ & $\begin{array}{l}(0.73, \\
0.34, \\
0.38)\end{array}$ & $\begin{array}{c}(0.64 \\
0.41 \\
0.47)\end{array}$ & $\begin{array}{c}(0.61, \\
0.41, \\
0.47)\end{array}$ & $\begin{array}{c}(0.68 \\
0.38 \\
0.43)\end{array}$ & $\begin{array}{l}(0.55, \\
0.50, \\
0.54)\end{array}$ & $\begin{array}{c}(0.57, \\
0.49, \\
0.53)\end{array}$ & $\begin{array}{l}(0.57, \\
0.49, \\
0.53)\end{array}$ & $\begin{array}{l}(0.51, \\
0.56, \\
0.59)\end{array}$ & $\begin{array}{l}(0.55, \\
0.51, \\
0.56)\end{array}$ & $\begin{array}{l}(0.53, \\
0.54, \\
0.58)\end{array}$ \\
\hline $0.5 Q^{(2)}$ & $\begin{array}{c}(0.36, \\
0.82, \\
0.79)\end{array}$ & $\begin{array}{c}(0.38, \\
0.81, \\
0.78)\end{array}$ & $\begin{array}{c}(0.34 \\
0.81 \\
0.78)\end{array}$ & $\begin{array}{l}(0.41, \\
0.68, \\
0.68)\end{array}$ & $\begin{array}{c}(0.28 \\
0.85 \\
0.84)\end{array}$ & $\begin{array}{l}(0.34, \\
0.75, \\
0.74)\end{array}$ & $\begin{array}{c}(0.32 \\
0.79 \\
0.77)\end{array}$ & $\begin{array}{c}(0.32 \\
0.79 \\
0.77)\end{array}$ & $\begin{array}{l}(0.31, \\
0.77, \\
0.76)\end{array}$ & $\begin{array}{l}(0.24, \\
0.85, \\
0.85)\end{array}$ & $\begin{array}{l}(0.34, \\
0.77, \\
0.75)\end{array}$ \\
\hline$Q$ & $\begin{array}{c}(0.74, \\
0.28, \\
0.32)\end{array}$ & $\begin{array}{c}(0.75, \\
0.27, \\
0.30)\end{array}$ & $\begin{array}{c}(0.67 \\
0.36 \\
0.37)\end{array}$ & $\begin{array}{c}(0.66, \\
0.28, \\
0.32)\end{array}$ & $\begin{array}{c}(0.69 \\
0.32 \\
0.37)\end{array}$ & $\begin{array}{l}(0.58, \\
0.38, \\
0.40)\end{array}$ & $\begin{array}{c}(0.59, \\
0.39 \\
0.41)\end{array}$ & $\begin{array}{c}(0.59, \\
0.39 \\
0.41)\end{array}$ & $\begin{array}{l}(0.54, \\
0.43, \\
0.45)\end{array}$ & $\begin{array}{l}(0.56, \\
0.43, \\
0.48)\end{array}$ & $\begin{array}{l}(0.57, \\
0.41, \\
0.44)\end{array}$ \\
\hline$S(Q)$ & 0.691 & 0.696 & 0.626 & 0.629 & 0.645 & 0.569 & 0.573 & 0.573 & 0.537 & 0.545 & 0.554 \\
\hline Rank & 2 & 1 & 5 & 4 & 3 & 8 & $6-7$ & $6-7$ & 11 & 10 & 9 \\
\hline
\end{tabular}


observation and is chosen as a next goal for the exploring autonomous robot.

Next, the case study results are discussed. As robot movement trajectories might be different in multiple simulations due to the various errors and incompleteness of robot sensor data (for example, artefacts in environment representation model, which is used for path planning), a total of 10 simulation runs were conducted for the PS, IG and WS strategies, and the averaged results of these tests are presented in Figure 3.

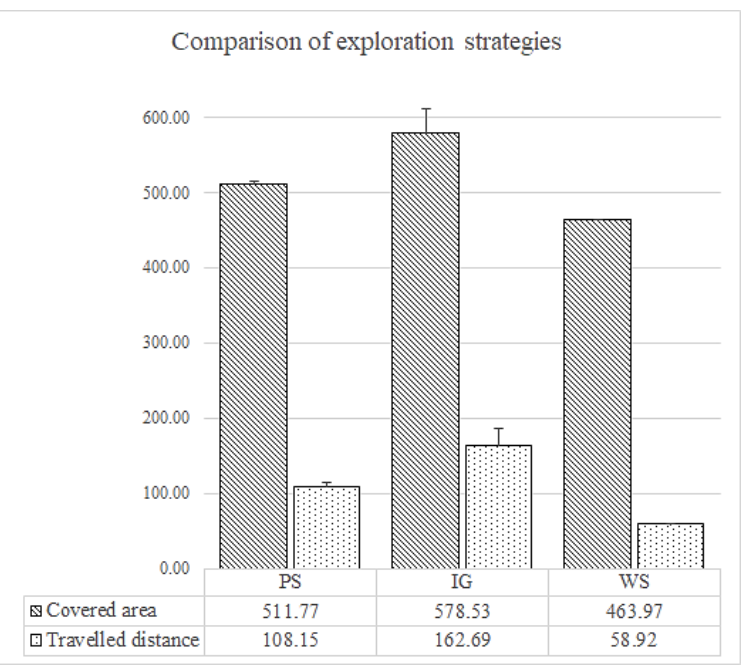

Figure 3. The average of covered areas in square meters and the total length of the robot travelled distance in meters after 10 simulation runs.

As it could be expected, on average, the WS strategy shows the best performance in the simulated environment when considering the average distance travelled by the autonomous robot. However, by applying this approach the robot acquires less information about the exploration environment when compared to the IG strategy and the proposed PS strategy. Considering the length of the robot travelled distance, the worst performance is observed when the IG strategy is applied. Although this strategy enables the robot to cover most of the exploration space, it also significantly increases robot backtracking and does not guarantee the visitation of all the priority locations. As such, the addition of $C_{5}$ and $C_{6}$ criteria shows potential in keeping the robot close to the prioritised locations while also minimising its chance to select the frontiers that are surrounded by largely explored space.
These results are also represented by the sample robot movement trajectory presented in Figure 4.

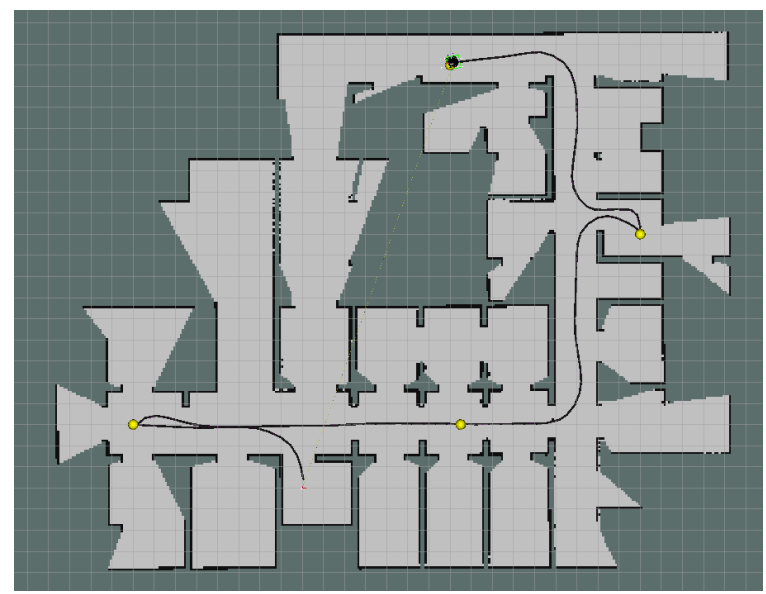

(a)

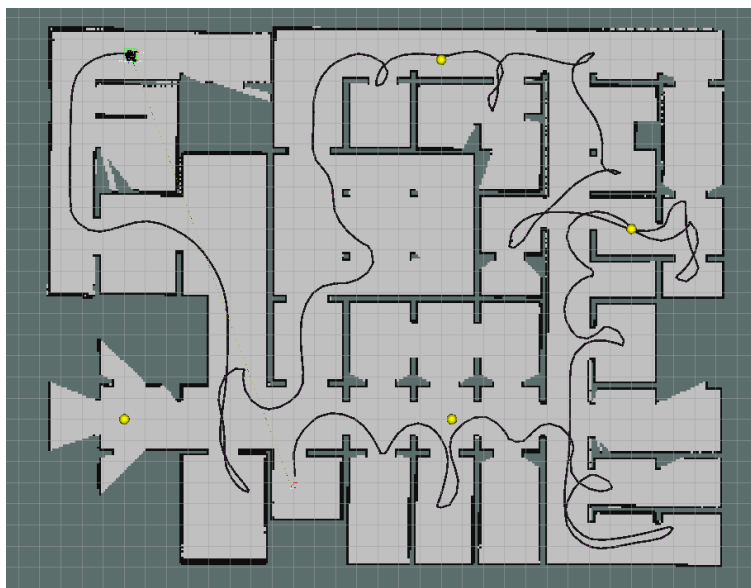

(b)

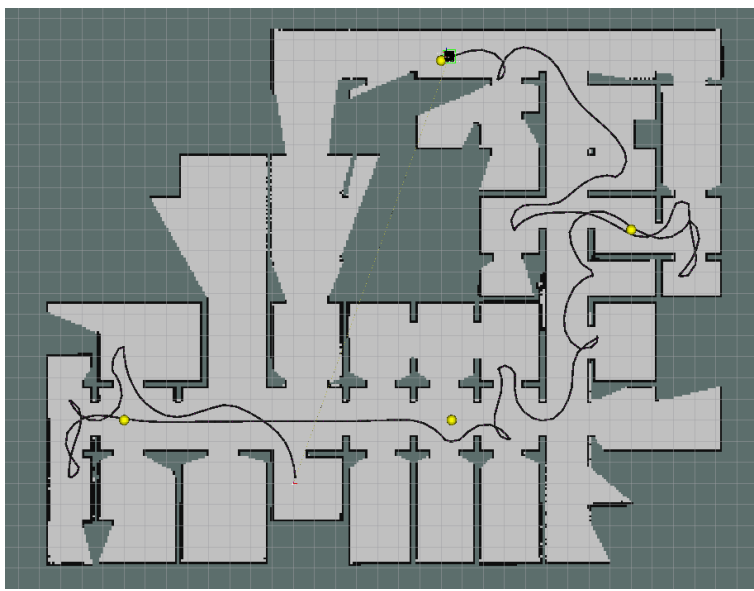

(c)

Figure 4. (a) - robot movement trajectory when the WS strategy is applied; (b) - robot movement trajectory when the IG strategy is applied; (c) - robot movement trajectory when the PS strategy is applied

For example, (a) showcases a sample of the robot movement trajectory when the WS strategy is 
applied. Here, the robot moves by following the shortest path between the set priority locations and finishes the exploration mission on an average of three minutes. On the other hand, it is common for the IG strategy to skip some of the priority locations and exhaustively explore the environment until the given time limit is reached and the robot is stopped, as highlighted in (b). However, the proposed approach, presented in (c), showcases that although this method covers less area when compared to the IG strategy, the robot is attracted to the priority locations, enabling it to explore the frontiers around these locations, and thus, detect more environment information (when considering the WS strategy) while also constructing time-efficient movement trajectory (when considering the IG strategy).

The obtained case study results lead to the conclusion that the proposed frontier assessment strategy enables the robot to maximise the covered area around the prioritised locations while maintaining a relatively short travel distance. However, it is worth noting that the actual robot behaviour mostly depends on the physical structure of the environment. In other words, in situations where the space around the prioritised location is cluttered or there is a lack of available frontiers, the robot might not be able to increase the amount of covered area before the task termination conditions are met.

\section{REFERENCES}

Akin, H. L., Ito, N., Jacoff, A., Kleiner, A., Pellenz, J. \& Visser, A. (2013). RoboCup Rescue Robot and Simulation Leagues, AI Magazine, 34(1), 78-86. DOI: 10.1609/aimag.v34i1.2458

Aruldoss, M., Lakshmi, T. M. \& Venkatesan, V. P. (2013). A Survey on Multi Criteria Decision Making Methods and Its Applications, American Journal of Information Systems, 1(1), 31-43.

Atanassov, K. T. (1986). Intuitionistic fuzzy sets, Fuzzy Sets and Systems, 20(1), 87-96. DOI: 10.1016/ S0165-0114(86)80034-3

Basilico, N. \& Amigoni, F. (2011). Exploration strategies based on multi-criteria decision making for searching environments in rescue operations, Autonomous Robots, 31(4), 401-417. DOI: 10.1007/ s10514-011-9249-9

\section{Conclusion}

Environment exploration by autonomous robots is a complex task that requires the balancing of competing high-level objectives. The additional complexity of such tasks stems from the uncertain and incomplete input data information applied in the decision-making process.

Although neutrosophic sets can be applied to model uncertain input data characteristics, a more generalised and flexible approach might be required in some complex decision-making tasks. Therefore, an extension of the state-ofthe-art WASPAS method modelled under the m-generalised q-neutrosophic environment is proposed, namely, WASPAS-mGqNS. An autonomous robot applied this novel method extension and the formulated frontier assessment strategy to balance the high-level objective requirements in autonomous environment exploration task. The case study results highlight how the proposed approach can enable the robot to cover more exploration space around the set priority locations while maintaining a relatively short movement trajectory. The obtained results also highlight that the proposed WASPAS-mGqNS extension could be applied to solve complex decision-making problems, such as iterative optimal frontier selection problems in autonomous environment exploration tasks.

Cakmak, F., Uslu, E., Amasyali, M. F. \& Yavuz, S. (2017). Thermal based exploration for search and rescue robots. In 2017 IEEE International Conference on INnovations in Intelligent SysTems and Applications (INISTA), (pp. 113-118). DOI: 10.1109/ INISTA.2017.8001142

Calisi, D., Farinelli, A., Iocchi, L. \& Nardi, D. (2007). Multi-objective exploration and search for autonomous rescue robots, Journal of Field Robotics, 24(8-9), 763-777. DOI: 10.1002/rob.20216

Chandrawati, T. B., Ratna, A. A. P. \& Sari, R. F. (2020). Path Selection using Fuzzy Weight Aggregated Sum Product Assessment, International Journal of Computers Communications \& Control, 15(5), 1-19. DOI: $10.15837 /$ ijccc.2020.5.3978

Juliá, M., Gil, A. \& Reinoso, O. (2012). A comparison of path planning strategies for autonomous 
exploration and mapping of unknown environments, Autonomous Robots, 33(4), 427-444. DOI: 10.1007/ s10514-012-9298-8

Roa-Borbolla, A. G., Marin-Hernandez, A., RechyRamirez, E. J. \& Vazquez-Leal, H. (2017). Priority exploration by mobile robots for search and rescue situations. In 2017 IEEE International Autumn Meeting on Power, Electronics and Computing (ROPEC), (pp. 1-6). DOI: 10.1109/ROPEC.2017.8261681

Semenas, R. \& Bausys, R. (2020). Modelling of Autonomous Search and Rescue Missions by IntervalValued Neutrosophic WASPAS Framework, Symmetry, 12(1), 162-183. DOI: 10.3390/SYM12010162

Smarandache, F. (1999). A Unifying Field in Logics: Neutrosophic Logic, Neutrosophy, Neutrosophic Set, Neutrosophic Probability, 1-141. American Research Press.

Smarandache, F. (2006). Neutrosophic set - a generalization of the intuitionistic fuzzy set. In 2006 IEEE International Conference on Granular Computing (pp. 38-42). DOI: 10.1109/ grc.2006.1635754

Smarandache, F. (2019). Neutrosophic Set is a Generalization of Intuitionistic Fuzzy Set, Inconsistent Intuitionistic Fuzzy Set (Picture Fuzzy Set, Ternary Fuzzy Set), Pythagorean Fuzzy Set, Spherical Fuzzy Set, and q-Rung Orthopair Fuzzy Set, while Neutrosophication is a Generalization of Regret Theory, Grey System Theory, and Three-Ways Decision (revisited), Journal of New Theory, 29, 1-31.

Taillandier, P. \& Stinckwich, S. (2011). Using the PROMETHEE multi-criteria decision making method to define new exploration strategies for rescue robots. In 9th IEEE International Symposium on Safety, Security, and Rescue Robotics, SSRR 2011 (pp. 321326). DOI: 10.1109/SSRR.2011.6106747

Wang, H., Smarandache, F., Zhang, Y. Q. \& Sunderraman, R. (2010). Single Valued Neutrosophic Sets, Multispace and Multistructure, 4, 410-413.

Yager, R. R. (2013). Pythagorean fuzzy subsets. In Proceedings of the 2013 Joint IFSA World Congress and NAFIPS Annual Meeting, IFSA/NAFIPS 2013, 2, (pp. 57-61). DOI: 10.1109/IFSA-NAFIPS.2013.6608375
Yager, R. R. (2017). Generalized Orthopair Fuzzy Sets. IEEE Transactions on Fuzzy Systems, 25(5), 1222-1230. DOI: 10.1109/TFUZZ.2016.2604005

Yamauchi, B. (1997). A frontier-based approach for autonomous exploration. In Proceedings of the 1997 IEEE International Symposium on Computational Intelligence in Robotics and Automation CIRA'97, "Towards New Computational Principles for Robotics and Automation", 146-151.

Zadeh, L. A. (1965). Fuzzy Sets. Information and Control, 8(3), 338-353. DOI: 10.1016/S00199958(65)90241-X

Zakiev, A., Lavrenov, R., Magid, E., Svinin, M. \& Matsuno, F. (2019). Partially Unknown Environment Exploration Algorithm for a Mobile Robot, Journal of Advanced Research in Dynamical and Control Systems, 11(8), 1743-1753.

Zavadskas, E. K., Bausys, R., Lescauskiene, I. \& Omran, J. (2020a). M-generalised q-neutrosophic MULTIMOORA for Decision Making, Studies in Informatics and Control, 29(4), 389-398. DOI: $10.24846 /$ v29i4y202001

Zavadskas, E. K., Bausys, R., Lescauskiene, I., \& Usovaite, A. (2020b). MULTIMOORA under Interval-Valued Neutrosophic Sets as the Basis for the Quantitative Heuristic Evaluation Methodology HEBIN, Mathematics, 9(1), 66. DOI: 10.3390/ math9010066

Zavadskas, E. K., Kaklauskas,A., Bausys, R., Naumcik, A. \& Ubarte, I. (2021). Integrated hedonic-utilitarian valuation of the built environment by neutrosophic INVAR method, Land Use Policy, 101(C): 105150. DOI: 10.1016/j.landusepol.2020.105150

Zavadskas, E. K., Turskis, Z., Antucheviciene, J. \& Zakarevicius, A. (2012). Optimization of Weighted Aggregated Sum Product Assessment. Elektronika ir Elektrotechnika, 122(6), 3-6. DOI: 10.5755/j01. eee.122.6.1810

https://www.sic.ici.ro 